\title{
Research on the Evaluation and Improvement of College Online Teaching Quality During COVID-19 Period_-Based on the Perspective of PDCA Theory
}

\author{
Hai-bo Yan ${ }^{\mathrm{a}}$, Wei $\mathrm{Li}^{\mathrm{b}} *$ \\ Academic Affairs Office, Jinan University, Guangzhou, China \\ a153812451@qq.com \\ b*627540195@qq.com
}

\begin{abstract}
Due to the influence of COVID-19, most colleges carried out online teaching. The evaluation of online teaching quality was the focus and difficulty of the sustainable development of online teaching. This research was based on the PDCA theory (Continuous Improvement Spiral), taking M University's online teaching during the COVID-19 period as a sample, and designing a questionnaire around the elements of online teaching quality in colleges. And then, we proposed a continuous improvement path for continuous improvement of online teaching quality evaluation in colleges from the various dimensions and levels of the quality evaluation results. This path is a "decision-operation-monitoring-improvement" closed-loop system, which provides external driving force for the operation of online teaching quality guarantee mechanism. We hope that this research can provide a reference for online teaching quality assurance in colleges.
\end{abstract}

Keywords: Online teaching, quality evaluation, PDCA, continuous improvement

\section{疫情时期高校在线教学质量评价与改进研究 基于 PDCA 循环理论的视角}

颜海波 $^{\mathrm{a}}$, 李伟 ${ }^{\mathrm{b}}$ *

\footnotetext{
暨南大学教务处, 广州, 中国

a153812451@qq.com

$b^{*}$ 627540195@qq.com
}

摘要: 疫情时期高校在线教学质量评价是在线教学可持续发展的重点与难点。本研究基于教学 质量持续改进 PDCA 循环理论的视角, 以 $\mathrm{M}$ 大学疫情时期在线教学为样本, 围绕疫情时期高校在 线教学质量的组成要素设计调查问卷, 结合在线教学质量评价结果, 探讨在线教学质量评价的 各维度与层面, 提出疫情时期高校在线教学质量评价的持续改进路径策略, 依托 “系主导主抓学院督促-学校抽检”、“教务处-学院” 对接联系的组织保障, 形成 “决策-运行-监控-改进” 闭环体系, 作为疫情时期高校在线教学质量评价的外驱系统, 为在线教学质量保障机制的运行 提供外驱力，以期为高校在线教学质量保障提供借鉴。

关键词: 在线教学; 质量评价; $P D C A$; 持续改进 


\section{1. 前言}

2020 年, 新型冠状病毒肺炎疫情爆发以来, 全 国各地启动一级响应。为阻断疫情向校园蔓延, 教 育部下发通知, 要求 2020 年春季学期延期开学, 按照 “停课不停教、停课不停学” 来备战疫情, 将 教学形式统一调整为在线教学, 突破以往时空的限 制。疫情的发生, 使得 “教师”与 “学生” 处于不 同的物理空间, 彼此分离, 以网络为媒介联系彼此。 在线教学的实施, 拓展了学生获取学习资源的途径, 改变知识习得方式，但是基于在线学习环境习得的 知识和技能无法和线下教学质量一致, 容易出现 “知识碎片化” “知识体系性不强” 等 ${ }^{[1]}$ 。现有相 关研究多关注疫情期间高校在线教学的过程研究, 而基于疫情特殊性的在线教学质量如何保障与提 高的研究并不多。而疫情时期在线教学质量的保障 与提高是关键, 也是需要关注的重点。基于此背景, 为探讨在线教学质量评价, 本研究结合 PDCA 循环 理论, 以 $\mathrm{M}$ 大学疫情时期在线教学为样本, 设计调 查问卷, 提出持续改进路径策略, 以期为在线教学 质量保障提供借鉴。

\section{2. 疫情时期高校在线教学质量内涵与 $\mathrm{PDCA}$ 循环理论的引入}

疫情时期高校在线教学质量评价有别于传统 教学质量评价方式, 其主要基于互联网这一载体, 综合运用现代信息技术开展教与学互动的新型教 育形态, 创新组织模式与教学模式, 采取多种教学 形式与手段, 开展在线教学活动的多元评价。在线 教学这一数字时代的新型教育生态系统, 学习者能 通过互联网随时随地学习数字化学习资源, 与教师 或其他学习者进行交互达成教学目标的一种学习 活动, 其核心要素包括教学目标、学习者、教师、 技术、交互、学习社区和学习资源 ${ }^{[2]}$ 。流畅的通信 平台、适切的数字资源、便利的学习工具、多样的 学习方式、灵活的教学组织、有效的支持服务、密 切的政企校协同是有效支撑疫情时期高校在线教 学的七个核心要素。[3]疫情时期在线教学一定程度 上转变了传统线下教学的师生角色关系, 重塑了传 统教学内容、变革了传统教学结构、再造了传统教 学流程、凸显了教学支持服务。在线教学质量评价 的核心因素, 主要是围绕教师、学生、教学内容与 数字化教学资源 (课程)、教学环境和多维教学评 价等方面展开。

20 世纪 50 年代初, 美国质量管理专家爱德 华. 戴明在全面质量管理工作中采用、普及并推广 PDCA 循环理论（又称 “戴明环” 或 “持续改进螺 旋”)。它是全面质量管理所应遵从的科学程序, 包括持续改进的四个循环反复的阶段: $\mathrm{P}(\mathrm{Plan})$-计划, $\mathrm{D}$ (Do)-执行, C(Check)-检查, A(Action)-处理, 被
广泛宣传、运用于持续改善产品质量的过程中。[4] 其特点是工作质量在不断循环中得到提高。引入 PDCA 循环理论, 作为疫情时期高校在线教学的质 量监控手段。采用 PDCA 全面质量管理的模式, 使 在线教学质量评价在计划、执行、检查与处理的四 个阶段中, 其中 $\mathrm{P}$ 阶段是在线教学质量评价的计划 与决策阶段, $\mathrm{D}$ 阶段是实施执行阶段, $\mathrm{C}$ 阶段为监 控阶段, A 阶段为总结与改进阶段。每个项目也可 应用 PDCA 理论, 完成新一轮循环改善, 不断的得 到总结与提升, 形成良好的循环式上升。通过 “决 策-执行-监控-改进” 四个环节的渐进循环, 实现 教学质量的不断优化和人才培养质量的持续改进 ${ }^{[5]}$ 。 教学质量仍是关键, PDCA 循环理论能为疫情时期 高校在线教学质量评价与改进提供思路和方法指 导。通过分析在线教学质量评价的结果, 为后续在 线教学质量评价提供持续改进的支撑。

\section{3. 在线教学质量评价的调查设计}

为充分了解疫情时期高校在线教学质量, 针对 全日制本科教学这一特定对象, 结合在线教学质量 的内涵, 分别设计教师、学生问卷。其中, 教师问 卷主要从教学理念、教学环境、教学内容、教学活 动 (教学形式和课堂互动) 、教学评价、教学效果、 整体满意度、困难及建议等方面展开; 学生问卷主 要从学习态度、学习预期、学习环境、学习资源、 学习活动 (教学形式和课堂互动)、教学评价、学 习效果、整体满意度、困难及建议等方面展开。教 师问卷、学生问卷分别围绕上述要素设置 19 个题 项。通过信效度分析, 信度系数值为 0.850 , 大于 0.8 , 说明数据信度质量高。针对 “CITC 值” , 分 析项的 CITC 值均大于 0.4 , 说明分析项之间具有良 好的相关关系, 同时也说明信度水平良好; 题项所 对应的共同度值均高于 0.4 , 说明题项信息可以被 有效提取。另外, KMO 值为 0.910 , 大于 0.6 , 意 味着数据具有良好的效度。

调查问卷, 面向全校参与本科教学的教师、全 日制本科生在线发放，共回收教师有效问卷 1318 份、学生有效问卷 8453 份。从教师参与调查情况 看, 无明显性别分布特征。年龄分布上, 参与调查 的教师大都集中在 35-45 岁之间, 占比 $42.79 \%$; 在 46-55 岁之间, 占比 30.96\%。职称分布上, 多集中 在 “副教授 (副高) ” 职称, 占比 $41.05 \%$; 课程多 集中在专业课, 占比 $75.8 \%$; 从参与调查的教师地 域分布来看, 有 $87 \%$ 的教师在广东。从学生年级分 布来看, 2019 级与 2018 级学生较为积极, 分别占 $33.08 \%$ 与 $27.71 \%$; 从性别分布来看, 女生比男生积 极, 女生占 $60.5 \%$, 男生占 $39.5 \%$, 也可能与 $\mathrm{M}$ 大 学学生性别比例有关。值得注意的是, 从参与调查 的学生分布来看, 超过 50\%的学生在广东省外。 


\section{4. 在线教学质量评价调查结果分析}

\section{1 重塑在线教学观念和学习态度是开展 在线教学质量评价的重要支撑}

参与调查的教师大部分表示在疫情发生之前 从未有过线上教学经历, 或者接触过线上教学的, 占比高达 $62.37 \%$; 有 $26.93 \%$ 的教师开展过其他形 式的线上线下相结合的混合式教学, 仅有 $10.7 \%$ 的 教师开展过慕课建设与应用, 以及运用慕课或线上 教学资源开展翻转课堂。在线教学的顺利开展, 对 教师的在线教学的信念与态度、信息化教学能力和 经验有着较高要求。由于在线教学经验的缺失, 大 多数教师对 “仓促上课” 的在线教学产生了一定 的知识焦虑和本领恐慌。但是大部分教师对疫情时 期 “停课不停学” 在线教学的认识都比较到位, 持 积极态度。50.15\%教师认为在线教学是顺利完成教 学计划的有效方式, $40.52 \%$ 的教师认为春季学期疫 情防控特殊阶段组织开展的在线教学是创新教学 方式方法的好机会。总体上看, 与传统线下教学相 比, 学生对在线教学态度较为积极, 仅有不到 $20 \%$ 的学生表示不喜欢在线教学; 绝大部分学生认为学 校 “停课不停学” 线上学习体验符合心理预期, 表 现出较好的自律性和自主性, 只有不到 $10 \%$ 的学生 认为不符合预期。

\section{2 优化在线教学环境、重构资源供给方 式是开展在线教学质量评价的有效载体}

各类教学工具和网络平台等教学环境能为在 线教学的有效运行和质量评价提供技术和网络优 势。作为在线教学运行重要载体和实现路径, 各种 在线教学平台协调好教师、学生及其各类软硬件条 件支持的关系，重构资源供给方式，利用大数据充 分释放在线教学平台的优势, 从管理上有效激发、 引导学生自主学习的主动性和能动性, 实现在线教 学平台的可获性, 帮助学生形成知识体系建构的能 力, 形成在线教学质量评价的有效载体。

数据显示, 师生广泛采用各种教学工具和教学 平台, 教师最喜欢的教学工具和平台是腾讯课堂/ 腾讯会议, 占比 $66.24 \%$, 其次是雨课堂, 占比 $48.41 \%$, 再者是社交工具 QQ 和微信等, 分别占比 $33.46 \%$ 与 $47.27 \%$, 喜欢采用中国大学 MOOC 的教师占比 $26.33 \%$ 。学生喜欢的教学工具和平台与教师的喜好 不完全一致。学生最喜欢的教学工具和平台也是腾 讯课堂/腾讯会议, 占比 $79.19 \%$, 其次是中国大学 MOOC, 占比 $63.1 \%$, 再者是雨课堂, 占比 $33.76 \%$ 。 结合上述数据, 师生能广泛采用各种教学工具和平 台, 最喜欢腾讯课堂/腾讯会议。值得注意的是, 分 别有 $20.64 \%$ 的教师、 $9.11 \%$ 的学生选择了其他教学 工具和平台, 通过词频分析, 教师们选用的 “其他”
类教学工具和平台主要集中在 ZOOM、钉钉、微助 教、Bilibili 等。

教学内容及学习资源供给方式的重构, 促使在 线教学质量评价的转变, 进一步优化教学内容, 凸 显课程思政育人元素。疫情期间学生在线学习可能 创生的附加价值包括对新冠病毒的认识、爱国情怀、 思辨能力、信息素养等, 这些都是学生创生的教学 目标以外的积极价值, 有助于学生的发展和成长。 为更好适应在线教学, 进一步提升教学质量, 大部 分教师对教学内容和教学资源进行了重新设计和 安排, 占比达到 $51.21 \%$; $38.62 \%$ 的教师对教学内容 作了微调, 仅有 $3.79 \%$ 的教师沿用传统线下教学的 教学内容, 未对教学内容做任何处理。学生对任课 教师准备的在线教学内容、教学资源的数量和质量 基本满意, 认为数量和质量都基本能满足学习需求, 占比 $62.07 \%$; 认为教学内容和资源数量和质量一般 的占比 $19.24 \%$; 对教学内容和数量非常满意的占比 $12.94 \%$ 。

\section{3 重塑在线教学课堂模式与教学流程是 开展在线教学质量评价的根本核心}

在线教学不是简单的线下教学的线上化, 而是 需要重塑课堂模式, 进一步优化教学流程, 丰富教 学形式, 强化课堂互动。进一步分析, 在线教学实 施过程包括 “怎么教” (教学形式)、“教什么” (教学内容) 和 “如何连接” (教学互动)。其中 教学形式与互动关系对在线教学质量评价和教学 效果有较大影响, 这是开展在线教学质量评价的核 心要点。直播式教学是师生最喜欢的在线教学课堂 形式。数据显示, 教师喜欢运用直播式教学占比 $54.7 \%$; 其次是多元协同式教学形式。多元协同式 中教师们普遍采用 “直播+慕课” 的形式。学生们 最喜欢的也是直播式教学方式 (占比 $41.38 \%$ ), 其 次是录播式, 再者是慕课/SPOC 学习为主的学习方 式。

师生互动关系是在线教学质量评价的有效要 素, 多重主体之间教学互动是否顺畅直接决定在线 教学质量。教师认为, 教学效果较好的教学形式与 互动方式组合有 “慕课/SPOC 应用为主” + “布置 学生线上分组讨论”、“多元协同式, 如 (慕课+ 直播) ” + “布置学生线上分组讨论” 等。“学生 自主学习”+ “签到打卡、投票等” 的教学形式与 互动方式组合教学效果是最差的。教师主要采用实 时互动, 大部分教师一节课线上教学与学生互动时 间多集中在 10-20 分钟之间。数据显示, 教师与学 生的线上互动方式主要为实时互动, 通过直播式视 频/语音, 社交工具文字/语音等进行实时互动, 分 别占比 $40.59 \%$ 与 $35.89 \%$ 。互动时间上， $36.42 \%$ 的 教师一节课的线上教学与学生互动时间在 10 分钟 以内, $33.76 \%$ 的教师一节课的线上教学与学生互动 在 20 分钟以内。 
学生最喜欢的互动方式为 “同步+异步” 互动, 但大部分学生认为最合适的互动时间在 10 分钟以 内。从统计结果看, 学生最喜欢的互动方式有两种, 一种是直播式视频/语音实时交流互动, 一种是自己 有问题给老师留言, 老师定时集中回复我的问题, 占比都为 $27.22 \%$ 。其次是社交工具文字、语音实时 互动占比 $21.92 \%$ 。综合分析, 学生喜欢的互动方式 教师在课程知识讲解时适当与学生有互动, 学生学 习消化后有问题再私下单独与教师进行异步互动。 学生认为学习效果最差的教学形式与互动方式组 合是“自主学习”+ “其他”, “其他”主要集中 在 “直播”、“弹幕” 等方面。从互动时间来看, 学生选择 “更喜欢老师讲授, 不想线上互动” 占比 $32.08 \%$, 选择互动时间 10 分钟以内的占比 $30.51 \%$ 。 由此可以看出, 学生其实并不太喜欢在线上课堂与 教师进行互动, 具体原因有待进一步研究和考证。 概而言之, 教师认为以慕课学习为主或多元协同式 教学形式和线上分组讨论的互动形式组合是教学 效果比较好的组合。直播、慕课与弹幕互动等形式 的组合是学生喜欢且学习效果好的组合。同时也发 现, 教师和学生均认为自主学习教学形式的教学效 果较差, 说明学生的自主学习能力有待进一步提高。

\section{4 创新评价方式是开展在线教学质量评 价的重要基础}

教师主要采用作业/作品互评在线教学评价方 式, 学生最喜欢在线测试。数据显示, 教师采用最 多方式是作业/作品互评, 占比 $29.97 \%$, 其次是在 线测试, 占比 $21.7 \%$, 再者是教师评价为主的评价 方式, 占比 $17.6 \%$, 综合评价占比 $16.01 \%$ 。深入分 析 “综合评价” ，教师采用最多的是 “打卡+作业 互评” “其他” 选项中, 出现最多的是 “课堂提问” 和 “布置作业” 的评价方式。学生认为最好的在线 教学评价方式是在线测试, 占比 $24.26 \%$, 其次是问 卷调查/投票等, 占比 $24.22 \%$, 再者是作业/作品互 评。14.42\%学生认为教师评价为主最好, $14.43 \%$ 学 生认为网络打卡评价方式最好。进一步分析 “综合 评价”，学生认为最好的是 “打卡+作业互评”,

“其他” 选项中, 出现最多的是 “布置作业” 的评 价方式。几乎全部教师会在课后布置作业或预习任 务。93.47\%的教师会在课后给学生布置作业或预习 任务, 仅 3.49\%教师课后对学生无任何安排和要求, 还有 3.03\%的教师选择 “其他” 安排, 具体主要包 括安排学生复习、巩固和拓展等。

教学目标达到度是影响在线教学质量评价效 果的重要因素。教师通过各在线教学平台提供的各 种交互数据, 比如作业完成率、视频观看率、学生 发帖率等, 来掌握学生的学习情况。从调查结果看, 教师对教学目标达成度相对乐观, 但学生认为知识 点掌握情况较为一般。 $49.17 \%$ 教师认为在线教学的 教学目标达成度在 $80 \%-100 \%$ 之间, $34.14 \%$ 教师认
为在线教学的教学目标达成度在 $60 \%-80 \%$ 之间。 $41.97 \%$ 的学生认为能掌握 60-80\%的知识点, $29.8 \%$ 的学生认为能掌握 30\%-60\%的知识点, $17.01 \%$ 的学 生认为能掌握 80\%-100\%的知识点。教师对在线教 学效果的预期与学生对自己知识点掌握情况的评 估结果不太一致, 说明在线教学的教学质量监控及 反馈有待进一步加强。对于未掌握的知识点, $57.68 \%$ 的学生通过自己上网查找学习资源的方式习得, 23.54\%的学生会通过回看老师的教学过程的方式 习得, 一定程度上, 能反映学生的自主学习能力较 强。

\section{5 优化在线教学组织管理是开展在线教 学质量评价的条件保障}

在线教学质量评价要关注以学习效果为中心, 并以此为出发点和落脚点, 获取更为直接的评价数 据。教师认为难以掌握学生学习状态和学习效果是 在线教学最大的困难所在。调查结果显示, 教师认 为在在线教学中遇到的最大困难是难以掌握学生 真实学习状态和学习效果, 占比 $40.29 \%$, 从前面教 师和学生对教学效果认识不一致也反映出这一点。 其次是很难与学生进行有效互动, 占比 $19.2 \%$, 再 者是网络教学环境、平台和工具等不稳定, 占比 $18.82 \%$, 备课时间远远超过传统教学, 占比 $12.59 \%$ 。 值得注意的是, 在 5.99\%教师选择的“其他”选项中, 很难营造教学氛围、缺乏面对面情感交流互动、无 法让学生进行实操等困难出现频次较高。与教师不 太一致的是, 学生认为在线教学最大的困难是网络 教学环境、平台和工具等不稳定, 占比 $27.64 \%$, 尤 其是在线教学在技术、资源选择和师生跨空间交互 上遇到的问题, 希望得到及时沟通交流。其次是学 习时间远远超过传统学习时间, 占比 $20.7 \%$, 再者 是不适应新的学习方式, 占比 $14.75 \%$ 。值得注意的 是, $6.7 \%$ 的学生选择了 “其他” 选项, 在该选项中, 在家学习氛围不浓、作业太多、自律自控能力不强、 线上学习容易走神、学习效率低、长时间线上学习 眼睛累等出现频次较高。

全校师生对学校及学院本次在线教学组织工 作比较满意。数据显示, 整体上, 教师和学生对学 校本次在线教学的总体安排、学校本次在线教学过 程中学院组织工作都比较满意。学校在在线教学的 技术支持、教学效果反馈、对学生关注关心等方面 有待进一步加强。在接下来的教学中, 教学支持服 务与教学效果的监控与反馈须重点改进。教师认为, 学校对在线教学的教学支持服务 (如技术支持) 有 待进一步加强, 占比 $41.35 \%$, 工作创新性也有待进 一步改善, 占比 $14.11 \%$ 。10.77\% 的教师认为培训工 作需要继续加强, 同时, $10.77 \%$ 的教师认为在给予 教师更多自主性和自由度、降低工作负荷等方面需 要进一步改善。在疫情发生之前, 在线教学非强制 性要求, 学校通过项目引导等多种方式, 但整体上 
教师主动开展在线教学的动力不足; 当在线教学面 临疫情非常时期, 成为不得不面对的现实难题时, 教师在线教学的支持服务和教学能力的提升则成 为当务之急。

\section{5. 疫情时期高校在线教学质量评价的持 续改进路径}

本研究基于教学质量持续改进 PDCA 循环理论 视角, 围绕疫情时期高校在线教学组成要素, 结合 在线教学质量评价结果, 提出疫情时期高校在线教 学质量评价的持续改进路径, 依托 “系主导主抓一 学院督促-学校抽检”、“教务处-学院” 对接联系 的组织保障, 可利用 PDCA 循环理论遵循 “P(计 划)-D(执行)-C(检查)-A(处理)” 的路径(详见图 $1^{[6]}$ ), 不断发现问题、解决问题, 形成 “决策-运行一监控 一改进” 闭环体系，作为疫情时期高校在线教学质 量评价的外驱系统, 为在线教学质量保障机制的运 行提供外驱力, 持续提升在线教学质量。

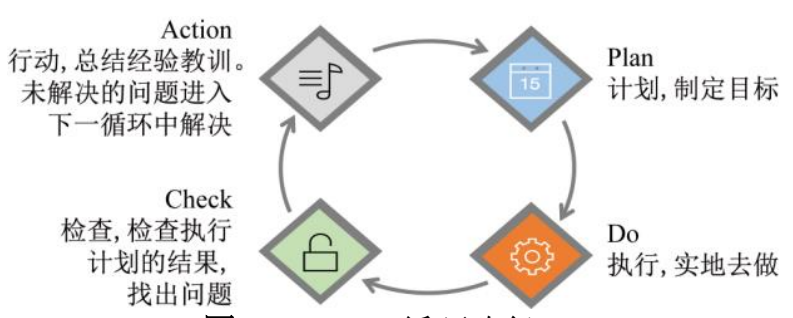

图 1 PDCA 循环路径

第一, 决策与计划。在线教学质量评价是一项 复杂的系统工程, 科学、精准的决策是疫情时期高 校在线教学质量评价有效运行的前提。决策主要包 括学校根据教育部及地方政策, 以学生为中心, 结 合学校、学生的实际情况提前部署制定的计划安排、 工作方案, 制定在线教学指南、发布在线教学通知 等。同时, 决策须与学校总体规划、统筹设计紧密 结合, 进一步完善组织保障, 在“系主导主抓-学院 督促-学校抽检”保障基础上, 建立“教务处一学院” 对接联系机制, 即建立教务处教学管理人员与各学 院的对接联系机制, 明确教学管理人员的对接学院、 对接任务和责任, 提高师生在线教学困难解决效率, 进一步提高在线教学质量。

第二, 运行与执行。计划决策的实施是实现在 线教学质量评价的前提条件。制度的生命力在于执 行, 在科学决策基础上, 制定有效、创新的措施是 教学质量保障机制平稳运行的关键。运行可分为教 学准备、教学运行、教学评价、支持服务四个部分。 教学准备包括根据疫情时期高校在线教学学生与 教师实际情况, 着眼于加强师生在线教学培训、统 计并公布教师在线教学形式、教务系统与各教学平 台对接、在线教学试运行等。教学运行主要指在线 教学正式运行, 即围绕教学目标开展各种教学活动, 包括鼓励教师采用多元协同在线教学形式、鼓励灵
活安排课程时间、多元活动等。教学评价鼓励教师 采取多元、多维评价, 关注学生学习生成和附加价 值。支持服务主要指加强技术支持, 及时多渠道多 方式答疑以及做好典型案例收集与推广等。

教学支持服务改进, 可设立学院教学平台管理 员。为了提高教学支持服务效率, 学校与在线教学 平台 (如雨课堂) 协同为每个学院设立了一名管理 员角色, 管理员可以及时为教师与学生提供技术支 持服务。统计确有学习困难的学生情况, 一对一帮 扶解决。部分学生居住境外与国内时差太大、居住 偏远山村网络环境太差等原因无法正常参与线上 学习, 基于此, 学校让每个学院每周统计并了解确 有学习困难的学生情况, 提出一对一帮扶解决方案, 以确保每个学生都能达成学习目标。

第三, 监控与检查。质量监控是基础, 是对在 线教学情况进行自我诊断的一种方式。检查是对在 线教学质量的查漏补缺, 可以发现前期工作中的种 种问题, 围绕在线教学涉及的各环节和要素开展质 量监控, 既是检验质量保障机制运行状况的重要途 径, 也是改进下一阶段在线教学的重要依据。在线 教学质量监控具体可从领导随机进入线上课堂听 课、在线教学数据抽查与监控、在线教学质量调查、 在线教学质量周报等方面进行, 能及时对执行过程 环节中存在的问题进行调整。

为进一步加强教学质量监控, 建立督导随机线 上听课制度, 组织教学督导随机进入线上云课堂听 课, 将听课情况反馈给教师, 可帮助教师改进教学。 到课率一定程度上反映课堂教学效果。建立本科课 程在线教学平均到课率每周一报机制, 要求各学院 将本科课程在线教学的到课率及时上报学校, 关注 并分析到课率低的课程, 提出持续改进措施。为进 一步规范在线教学活动, 加强在线教学纪律管理, 建立健康有序的在线教学纪律。学校发布通知倡导 师生遵守课堂教学纪律, 要求教师积极弘扬社会主 义核心价值观, 严守师德师风, 严肃网络言行规范, 学生端正学习态度, 严以律己, 保质保量完成学习 任务。要求学生主动维护教师个人的知识产权。

第四, 改进与处理。改进与调整就是对前面监 控环节的信息进行处理并做出有效反馈, 并决定着 整个闭环是否能够顺利进行循环、如何循环、循环 的上升方向。持续改进机制是在线教学质量监控的 目的, 学校应针对当前在线教学过程中已经发现的 问题、薄弱环节以及可能会出现的问题, 建立行之 有效的改进和预防措施。具体可从优化在线教学质 量管理决策、优化在线教学运行、优化在线教学质 量监控、制定在线教学突发状况紧急预案等方面进 行。对于在线教学来讲, 建立在线教学与管理的大 数据反馈分析机制尤为重要。

转变理念，从“管理” 到 “服务”，充分运用 大数据技术, 实现个性化教学支持服务, 为在线教 学质量持续改进提供服务保障。“一刀切” 固化的 教学管理模式并不适合疫情期间时空分离的在线 教学, 在线教学最需要的保障是教学支持服务, 教 
师与教学管理者都应从教学管理转变为教学支持 服务者, 为教师和学生提供个性化教学支持服务, 精准保障教学效果。通过收集、监测、分析在线教 学平台上师生留下的教学数据, 科学、全面地从多 维度精准分析师生教与学状态和效果, 深度挖掘数 据背后的深层关系与隐藏价值，进而构建个性化教 与学支持服务系统、咨询与反馈服务系统等, 强化 个性化教学支持服务, 提升教学效果, 也为教育教 学相关政策制定提供数据支持。

综上所述, 将 PDCA 循环理论与在线教学质量 评价相结合在一定程度上能提升在线教学成效。在 对全校本科课程在线教学平均到课率的监测中, 总 体平均到课率在稳中不断向好。疫情虽然 “架空” 了实体学校, 后疫情时代, 学生开始强烈渴望回归 学校, 这似乎一定程度上证明实体学校的不可取代 性。虽然此次疫情让我们看到了在线教学的优势, 但传统教学的必要性无法取代。诸如, 返校后大部 分教师愿意开展线上线下混合式教学, 占比 $53.42 \%$; 其次是实体课堂教学占比 $43.34 \%$ 。然而, 混合式教 学融合传统教学与在线教学优势, 将是未来比较有 潜力的一种教学模式。这在一定程度上说明疫情时 期高校在线教学模式具有可持续性。积极推动混合 式教学探索与实践, 推动互联网与信息技术与教育 教学的融合与创新, 能为在线教学质量持续改进注 入新活力。接下来如何提升、监控在线教学质量, 可以从深化线上课堂互动、多元化教学评价、提供 精准教学支持服务保障等方面持续改进。因此, 借 助疫情时期在线教学惯性, 积极研究、推动混合式 教学探索与实践, 将是创新模式、推动教育教学改 革、构建教育教学新生态的重要路径。

\section{项目基金}

本文为广东省教育科学 “十三五” 规 划 2020 年度 “强师工程” 项目《粤港澳大 湾区高校在线开放课程多元协同开放应用 共享研究》（2020WQYB001）的阶段性成果 之一。

\section{REFERENCES}

[1] Xiulin Ma, He Mao and Cuixia Wang. (2016) Video types and their effect on learners'online learning experience: an empirical study. Distance Education In China, (4):32-39.

[2] Singh, Thurman. (2019) How Many Ways Can We Define Online Learning? A Systematic Literature Review of Definitions of Online Learning (1988-2018). American Journal of Distance Education, 33(4):289-306.

[3] R. H. Huang, M. H. Zhang, Y. Shen, Y. Tian, H. J. Zeng. (2020) Research on the Core Elements of Hyperscale Internet Education Organizations-Online Education Effectively Supports Case Analysis of Universities in Epidemic Situation. E-education Research, 41(3):10-19.

[4] James R. Evans \& William M. Lindsay. (2010) Quality Management and Quality Control (Seventh Edition). China Renmin University Press, Beijing.

[5] Z. G. Li, H. Wang, Q. Chen. (2018) A System of Teaching Quality Assurance for University Based on PDCA Model. Higher Education Development and Evaluation, 34(02):32-40+104.

[6] Ge S.J, Yang J . (2001) Modern Enterprise Management, Economic \& Management Publishing House, Beijing. 